\title{
Rationale for Possible Targeting of Histone Deacetylase Signaling in Cancer Diseases with a Special Reference to Pancreatic Cancer
}

\author{
Mehdi Ouaïssi, ${ }^{1,2,3}$ Urs Giger, ${ }^{4}$ Igor Sielezneff,, ${ }^{1,2}$ Nicolas Pirrò,,${ }^{1,2}$ Bernard Sastre,,${ }^{1,2,3}$ \\ and Ali Ouaissi ${ }^{5,6}$ \\ ${ }^{1}$ Service de Chirurgie Digestive Pôle d'Oncologie et Spécialités Médico-Chirurgicales, Hôpital Timone, \\ Assistance Pubique-Hôpitaux de Marseille, Marseille, France \\ ${ }^{2}$ Faculté de Médecine, Aix Marseille Université, Marseille, France \\ ${ }^{3}$ CRO2-INSERM, UMR911, Marseille, France \\ ${ }^{4}$ Department of General Surgery, Kantonsspital Liestal, 4800 Zofingen, Switzerland \\ ${ }^{5}$ INSERM, CNRS UMR 5235, Montpellier, France \\ ${ }^{6}$ IBMC, Universidade do Porto, 4009-002 Porto, Portugal \\ Correspondence should be addressed to Mehdi Ouaïssi, mehdi.ouaissi@mail.ap-hm.fr \\ Received 10 June 2010; Revised 9 September 2010; Accepted 23 September 2010 \\ Academic Editor: Christian Seiser
}

Copyright () 2011 Mehdi Ouaïssi et al. This is an open access article distributed under the Creative Commons Attribution License, which permits unrestricted use, distribution, and reproduction in any medium, provided the original work is properly cited.

There is ongoing interest to identify signaling pathways and genes that play a key role in carcinogenesis and the development of resistance to antitumoral drugs. Given that histone deacetylases (HDACs) interact with various partners through complex molecular mechanims leading to the control of gene expression, they have captured the attention of a large number of researchers. As a family of transcriptional corepressors, they have emerged as important regulators of cell differentiation, cell cycle progression, and apoptosis. Several HDAC inhibitors (HDACis) have been shown to efficiently protect against the growth of tumor cells in vitro as well as in vivo. The pancreatic cancer which represents one of the most aggressive cancer still suffers from inefficient therapy. Recent data, although using in vitro tumor cell cultures and in vivo chimeric mouse model, have shown that some of the HDACi do express antipancreatic tumor activity. This provides hope that some of the HDACi could be potential efficient anti-pancreatic cancer drugs. The purpose of this review is to analyze some of the current data of HDACi as possible targets of drug development and to provide some insight into the current problems with pancreatic cancer and points of interest for further study of HDACi as potential molecules for pancreatic cancer adjuvant therapy.

\section{Background}

Cancer diffusion and metastasis account for approximately $90 \%$ of all cancer-related deaths [1]. Metastasis follows a multistep complex processes in which neighboring healthy tissue is invaded by primary tumor cells, which access the systemic circulation and finally proliferate at distant sites into macroscopic secondary tumors via the perivascular and/or perilymphatic tissue [2]. In the case of pancreatic cancer, most of the patients already have metastases at the time of diagnosis. These patients have a poor prognosis, and less than $5 \%$ of patients are alive 5 years after the initial diagnosis [3].

Specific events that promote tumorigenesis and cancer progression are linked with complex molecular modifications such as DNA methylation, histone acetylation, phosphorylation, ubiquitylation, and ADP ribosylation. Currently, results from basic research underline the importance of acetylation and deacetylation at the level of not only histone lysine residues but also other cellular factors that are supposed to interfere with the regulation of gene expression. HDAC enzymes play a central role to oppose histone deacetylases (HDATs), which catalyze histone acetyltransferases (HATs) [4].

Previously, eighteen mammalian HDACs have been characterized and are currently classified as follows: Class I and II share similarities with yeast deacetylases RPD3 and HDA1; Class III shows homology to yeast silent information regulatory protein (SIR2p) [5]; Class I includes HDACs 1, 2, 3, and 8; Class II comprises HDACs 4, 5, 6, 7, and 9 [5]. HDAC6 and HDAC10 are carriers of two catalytic sites 
and are therefore grouped in subclass IIB. HDAC 11 shares conserved residues with Class I and II enzymes in their catalytic site and is allocated to Class IV [5].

Based on their primary structure, the SIR2 family [Hst proteins (Homologous of Sir two)] or sirtuins are currently grouped into five different classes [6]: Sirtuin Class I: Human SIRT1, 2, 3; Class II: SIRT4; Class III: SIRT5; Class IV: SIRT6, 7. SIR-T8, which was recently detected in thyroid carcinoma cell lines and tissue samples [7], shares $85 \%$ homology in the core sirtuin domain with SIR-T7 and is therefore grouped into Class IV.

HDAC enzymes differ in their subcellular localization, catalytic activity, and susceptibility to different inhibitors. Class I HDACs are found exclusively in the nucleus, whereas HDAC3 has both nuclear import (NIS) and export (NES) signals being able to localize to the cytoplasm [8]. The absence of NES in HDAC1 and HDAC2 sequences attest to their nuclear localization [8]. HDAC11, the unique member of class IV, resides in the nucleus [5]. While Class II HDACs are able to shuttle in and out of the nucleus, the Class III sirtuin family (SIRT1-7) has a different localization. Whereas three SIRT proteins (SIRT1, SIRT6, and SIRT7) are nuclear localized, SIRT3, SIRT4, and SIRT5 are localized in the mitochondria and SIRT2 is a cytoplasmic protein [8].

HDAC enzymes deacetylate histones and other protein substrates. Trichostatin (TSA), a fermentation product of Streptomyces, originally used as an antifungal agent, was found to have anticancer cells proliferation activity and the ability to inhibit HDAC with an $\mathrm{IC}_{50}$ in the nanomolar range [8]. In a recent elegant study a high-throughput, precise profiling of HDACi potency against all class I and II enzymes has been achieved using a panel of structurally diverse small HDACi molecules comprising those reported in the literature [9]. Surprisingly, an apparent redundancy of pharmaceutical compounds toward HDAC1, HDAC2, and HDAC3 was evidenced and at relevant concentrations the class IIa enzymes are not targeted by most HDACi tested. Unlike other class I and II HDACs, the sirtuins require nicotinamide dinucleotide (NAD) as a cofactor. The search for sirtuin inhibitors has identified, besides the physiological inhibitor nicotinamide, synthetic inhibitors such as sirtinol and splitomicin $[10,11]$.

\section{HDACs and Cancer}

Growing knowledge about HDACs/SIRTs shows that they are regulators of growth, differentiation and cell death (apoptosis). The dysfunction of transcriptional repression mediated by HDACs may lead to carcinogenesis. Indeed, modulation of expression levels of genes encoding HDACs (over- and/or underexpression) has been reported for different types of cancer [5]. For example, overexpression of HDAC1 has been reported in gastric [12] and HDAC2 and HDAC3 in colorectal cancer $[13,14]$. Decreased transcription of the HDAC5 gene has been observed in colorectal cancer [15]. In regards to Class III HDACs, SIRT8 was found to be overexpressed in thyroid cancer [16], while SIRT2 gene expression is downregulated in human gliomas [17].
Among the targets of HDACs are members of the family of nuclear factors Rel/NF-kappa B. However, NF-kappa B is activated in the early stages of tumor transformation of mammalian cells [18]. Similarly, NF-kappa B is constitutively active in the case of human adenocarcinoma of the pancreas [19] and in leukemic T-lymphocytes [20], but not in breast cancer [21].

It has also been demonstrated that the action of HDACs may modulate the activity of NF-kappa B. Indeed, deacetylation of the RelA/p65 subunit of NF-kappa B by HDAC1, HDAC2 [22], or HDAC3 [23] increases its association with $\mathrm{I} \kappa \mathrm{B} \alpha$, which leads to a loss of transactivation activity. Furthermore, deacetylation of lysine 310 of Rel/p65 by SIRT1 represses the transactivating activity of $\mathrm{NF} \kappa \mathrm{B}$ and consequently its antiapoptotic property [24]. In summary, these data underline a possible involvement of HDACs in the process of tumorigenesis.

\section{HDACi and Cancer Therapy/Mechanisms of Action of HDACi}

Since the discovery of the anti-tumor effect of Trichostatine TSA in 1990 [25], many other HDACi have been identified. A major therapeutic limitation of HDACi is their nonselectivity. Indeed, they target both HDAC Class I and Class II. Although some HDACi have some degree of selectivity such as the depsipeptide (FK228) with a preference for HDACs 1 and 2 [9], to date, an HDAC isoform selective inhibitor has not been developed which indicates that selectivity may be less of a therapeutic limitation than the so-called pan-HDACi. The mechanism of action was elucidated by crystallization of the catalytic domain of TSA or suberoylanilide hydroxamic acid (SAHA) [26]. TSA is a product based on a hydroxamic acid, which reaches the active site of HDAC, chelates $\mathrm{Zn2+}$, and inhibits the enzyme at nanomolar concentrations $[5,26]$.

\section{Cytostatic Effect and Induction of Differentiation}

HDACi induce differentiation of tumor cells. Initial applications have focused on promyelocytic leukemia, in which the expression of the fusion protein PML-RAR plays a crucial role. This part of the RAR (retinoic acid receptor) behaves as a constitutive transcriptional repressor of differentiation; HDACi, which lift the repression, initiate the differentiation program. Similar results were obtained in various models of leukemia and solid tumors. The treatment of such tumors by a variety of HDACi shows that the inhibitors have a cytostatic effect on tumor cells by induction of cell cycle arrest in G1 or in some cases in the G2 phase [27]. The $p 21$ gene is the target that appears to be most often associated with this phenotype. It is a repressor complex that is formed by cyclins and kinases, cyclin E-Cdk2, and cyclin A-Cdk2 whose constitutive activity is responsible for the deregulation of the cell cycle of tumor cells. HDACi may act, at least in some cases, in synergy with retinoic acid, thereby inducing differentiation of myeloid cells in vitro and in vivo [5]. 


\section{Induction of Apoptosis}

In most cases, HDACi induce death of tumor cells by apoptosis through either the intrinsic or extrinsic pathways [27]. The intrinsic pathway involves the activation of mitochondrial proapoptotic members, which leads to the generation of free radicals. A number of studies have shown that overexpression of Bcl-2 not only inhibits apoptosis, but also inhibits the apoptosis-inducing effect of HDACi. For instance, lymphoma B cells that overexpress Bcl2 were found to be resistant to SAHA [5]. Moreover, in prostate cancer cell line PC3, SAHA causes an increase accumulation of Bcl-2 which correlates with the resistance to SAHA-induced cell death. In contrast, the lack of Bcl-2 in DU145 cell line is associated with its marked sensitivity [28]. In the extrinsic pathway, HDACi can induce expression of Fas and its ligand. The protein death domain TRAIL also seems to be induced in tumor cells derived from acute promyelocytic leukemia [29]. Indeed, in a transgenic mouse model defective in TRAIL receptor expression, treatment with valproic acid (HDACi of Class I) induces overexpression of these receptors. Moreover, their loss reduced the induction of apoptosis by $50 \%$ in the transgenic mouse model [30]. Additionally, it has been shown that HDACis have an effect on the cleavage of Bid and phosphorylation of Bad and Bim [31-33]. However, the initiation process is not yet well established. It is possible that Bid is activated via a promoter hyperacetylation and the activation of a transcription factor (E2F1) [34, 35].

\section{Effect on the Production of Oxygen Radicals}

HDACis induce the production of free oxygen radicals. However, the exact mechanism leading to this phenomenon is unknown [36]. A change of the mitochondrial membrane potential occurs after a rise in free oxygen radicals. However, even within this apoptotic pathway, there are a number of unknown parameters. Indeed, the work of Garcia Morales et al. [37] showed that despite overexpression of caspase 3 after treatment of three pancreatic cancer cell lines with HDACi, the inhibition of caspase 3 did not abolish cell death by apoptosis. These results support the fact that the apoptotic pathways induced by HDACi is still far from being elucidated.

\section{Antiangiogenic Activity}

The HDACi have antiangiogenic properties due to their ability to repress the expression of VEGF (vascular endothelial growth factor) in vitro and in vivo [38]. They also suppress the transcription of the chemokine (CXCR4) encoding gene that plays a pivotal role in both the differentiation of endothelial cells in the bone marrow and their mobilization [39]. Moreover, inhibition of HDACs induced by FK228, a cyclic depsipeptide isolated from Chromobacterium violaceum, increases gene transcription encoding factors that inhibit angiogenesis and decreases the expression of the gene encoding factors stimulating angiogenesis. These observations demonstrate that HDACis interfere with the process of neovascularization, which is pivotal for tumor growth.

\section{Immunomodulatory Properties}

HDACis play a role in anti-tumor immune mechanisms. HDACis activate the transcription of genes encoding MHC Class I and II molecules and also costimulate CD40, CD80, and CD86 molecules [40,41]. This biological property appears to be related to the removal of repression [42]. Furthermore, HDACi can also alter the secretion of different interleukins. For instance, suberoylanilide hydroxamic acid (SAHA) reduces acute graft-versus-host disease after allogenic bone marrow transplantation [43]. This action results from a decreased production of different interleukins (TNF, INF $\gamma$ and IL-1), which all play a major role in graftversus-host reactions. Moreover, it has been shown that the acetylation status of STAT1 (signal transducer and activator of transcription) [44], STAT3 [45], as well as NF-kappa B, is related to their degree of activation/deactivation, which affects the cytokine secretion profile [23]. Furthermore, SAHA activates differentiation of dendritic cells [46], which potentiates the immune response against tumors.

A link between inflammation and cancer development has been shown in population studies. Regulatory factors such as NF-kappa B, as described above, are substrates of HDACs [27]. In premalignant cells, NF-kappa B, by inducing the expression of proinflammatory and survival genes and inhibiting those that promote cell death, may increase the potential of precancerous cells. By blocking the transactivation of NF-kappa B, HDACi may promote the expression of genes that are repressed in tumor cells. Therefore, they exert their therapeutic effect by reverting the tumor phenotype of cancer cells to a normal phenotype.

\section{Combination of HDACi and Anticancer Drugs}

Interestingly, $\mathrm{HDACi}$ can potentiate the anti-tumor effect of drugs [27]. It has been previously demonstrated that coincubation of K562 leukemic cells with a proteasome inhibitor, bortezomib, SAHA, or sodium butyrate, increases apoptosis by inducing alteration of mitochondrial function [47]. Bortezomib, which is used for the treatment of myeloma, has a limited action due to formation of aggresomes and corresponding accumulation of ubiquitylated proteins. This limiting step is controlled by HDAC6. A recent study demonstrated that the use of TSA, which inhibits the action of HDAC6, enhances bortezomib-induced apoptosis in pancreatic cancer cells [48]. Moreover, pretreatment of human leukemic cells with MS-275, an orally active synthetic benzamidine derivative that belongs to HDACi significantly enhances the activity of the cytotoxic drug Fludarabine, a purine analogue that has demonstrated significant activity in B-cell malignancies [49].

\section{Pancreatic Cancer}

Pancreatic ductal adenocarcinoma (PDAC) is ranked fourth and fifth to sixth leading cause of cancer death in USA and Europe, respectively [50]. Standard treatments for 
advanced disease include radiotherapy and/or chemotherapy regimens. However, radiotherapy is often toxic and the chemotherapy which includes drugs such as 5-fluorouracil (5-FU) and gemcitabine (GEM) are either ineffective or effective for only short duration [51]. Recent phase III trial comparing the efficiency of treatment with either GEM alone or GEM combined to Erlotinib, an inhibitor of EGFR tyrosine kinase showed a modest increased survival time of 15 days [52]. Furthermore, a combination of GEM with the bevacizumab, an inhibitor of VEGF, in a phase III trial did not increase survival time [53]. Moreover, a failure to demonstrate significant clinical benefit for the patients was recorded in a phase III trial combining GEM and cetuximab, an anti-EGRF monoclonal antibody [54].

Historically, the ras oncolo-proteins have been considered the target substrate responsible for the antiproliferative effects of enzyme farnesyl protein transferase (FPT) inhibition. Despite 70 to $90 \%$ mutation of K-ras in pancreatic cancer [55], the combination of FPT inhibitor and gemcitabine didnot prolong overall survival in advanced pancreatic cancer compared with single agent gemcitabine [56]. The raisons for the failure of these treatments are not yet understood.

Tumor chemoresistance could be among factors responsible for the lack of effective therapies [57]. Indeed, multiple biochemical and molecular alterations occur in cancer cells. For instance, pancreatic cells overexpress the death receptor decoys DcR2 and DcR3 as well as BcL-XL which play a role in pancreatic cancer chemoresistance [57]. Moreover, a number of other genetic alterations for several specific genes including $p 53, p 16^{I N K 4 a}$, and Smad4, have been documented [58]. Interestingly, reintroduction of the wild-type p53 in the human pancreatic cells increases their sensitivity to GEM [59].

Therefore, in pancreatic cancer, there is an urgent need for rationally designed molecules displaying improved efficacy and tolerability compared to existing treatments. HDACi have emerged as promising antineoplastic agents. In human pancreatic adenocarcinoma cell lines, TSA and SAHA have been shown to induce the cell death by apoptosis found to be caspase independent [60]. Other investigators have reported that TSA can synergize with gemcitabine [61] or the proteasome inhibitor PS-341 [62] to induce apoptosis of human pancreatic cancer cells. In a recently published study by our research group, we demonstrated that HDAC Class I and II inhibitors such as TSA can induce death of tumor cell lines via apoptosis [63]. Interestingly, the data also showed, for the first time, that Class III HDACis, such as sirtinol and nicotinamide, are able to induce pancreatic cell death. However, whether sirtuin inhibitors are efficient anticancer drugs in vivo is yet to be demonstrated.

To provide insight into the biological behavior of pancreatic cancer and to identify new potential biomarkers, a study aiming to examine the levels of HDAC and SIRT gene expression in pancreatic cancer compared to normal pancreas tissue has been initiated. Because normal pancreatic tissue can only be obtained under certain circumstances (i.e., donor liver transplantation), samples of control tissues from the surgical specimens of patients with pancreatic adenocarcinomas [close proximity termed normal adjacent, far away from the tumor as possible termed normal distant], serous cystadenoma (SC), intraductal papillary mucinous tumor of the pancreas (IMPN), or complicating chronic pancreatitis (CP) have to be used as controls. So far, an increased number of SIRT5 mRNA transcripts have been observed not only in most of the Pancreatic adenocarcinoma (PA) samples but also in other tissues samples (SC, IMPN and $\mathrm{CP}$ ). Approximately $81 \%$ of the PA tissue samples displayed increased expression of HDAC7 mRNA transcripts as well as its corresponding protein [64]. Furthermore, it has been shown that more than $90 \%$ of the analyzed PA samples contain activated point mutations of the K-ras gene, and a large number of these neoplasms also exhibit alterations in genes controlling the G1/S-phase cell cycle transition such as $p 16_{I N K 4 a}$ [65]. Additionally, the $p 16_{I N K 4 a}$ genetic alterations are significantly more frequently observed in patients with the shortest tumor survival compared with those patients with the longest [65]. Other molecular alterations may contribute to carcinogenesis of the pancreas such as those related to growth factors and/or their receptors. Due to the fact that almost $90 \%$ of the patients are unsuitable for resection, our own data are the first to demonstrate that HDAC gene expression, particularly HDAC7, could be a possible marker of PA [64]. Although it is difficult to determine at this stage whether upregulation of HDAC7 in $\mathrm{PA}$ is a cause or a consequence of malignant progression, its overexpression in cancerous tissues and not in their normal counterparts constitutes an interesting field of future research for new approaches in the design of antipancreatic cancer therapy.

HDACs are key modulators of endothelial cell migration and angiogenesis and regulate PDGF-Bp/PDGF-beta gene expression [66]. Taking into account that angiogenesis is required for tumor progression, it is reasonable to suggest that molecules that are able to interfere with HDAC expression/activity may be of particular therapeutic benefit. In this perspective, a recent study has shown that vorinostat, the first HDACi approved for clinical trials in the treatment of cutaneous T-cell lymphoma [67], selectively downregulates HDAC7 expression [68]. However, rational targeting of HDAC7 signaling will require extensive investigation of cross-talk between the HDAC7 signaling pathway and other pathways involved in pancreatic cancer progression. This will probably allow us to define which component will be more effective when combined with a potential HDAC7 inhibitor. Another field nicely developed in a recent review by Stimson and La Thangue is the search for predictive biomarkers that may inform on the tumor response to HDACi [69]. For instance, the $H R 23 B$ gene validated as a sensitivity determinant for HDACi-induced apoptosis, was identified at high levels in cutaneous T-cell lymphoma (CTCL) in situ, a malignancy that responds favorably to HDACi-based therapy. Therefore, the identification of such biomarkers which might be linked to HDAC7 overexpression will help to select patients who might benefit from HDACi therapy. A recent study have shown that histone modification levels indicate patients treated for pancreatic cancer whith adjuvant chemotherapy were more or less likely to derive survival 
benefit from adjuvant fluorouracil relative to gemcitabine. Although differences were modest and require validation, they raise the possibility that histone modification levels could serve as predictive biomarkers for adjuvant treatment [70]. For instance, it has been previously demonstrated that HDAC7 interacts with the transcriptional regulator MEF2D, which binds to the promoters and transcriptionally represses the proapoptotic orphan receptor Nur77 [71]. Interestingly, treatment of CTCL with panobinostat (LBH589, Novartis Pharmaceuticals Basle, Switzerland) inhibits the mRNA and protein levels of HDAC7 and induces expression and translocation of Nur77 to the mitochondria where it converts death resistance protein BCL-2 into a killer protein, therefore leading to the death of cultured and patient-derived human CTCL cells [72]. Furthermore, the majority of HDAC7 are localized in the nucleus; however, despite the absence of NES signal, it is also found in the cytoplasm. The regulation of nucleocytoplasmic shuttling is not yet clearly defined, although studies have shown that the cellular concentration of factors such as 14-3-3 (a cytosolic anchor protein), CaMK I (Ca2+/calmodulin-dependent kinase), and other yet unknown molecules may determine the subcellular localization of HDAC7 in a cell type and HDAC-specific manner [73]. Others have reported that the activity of HDAC7 in the nucleus is dependent upon its interaction with HDAC3. Consequently, cytoplasmic HDAC7, which is not bound to HDAC3, is enzymatically inactive [74]. Taken together these observations pointed to the need of further investigations of pancreatic-tumor-associated HDAC7 in order to better understand its exact role in cancer progression and to design selective inhibitors containing a variety of scaffolds with interesting physicochemical properties.

\section{Critical Point of View}

The critical challenge in pancreatic cancer is the detection of early lesions at an asymptomatic stage that will allow for curative resection and to offer a greater chance of a cure. In recent years, HDACs emerged as promising targets for therapeutic interventions that could revert the aberrant epigenetic states associated with cancer $[75,76]$. Thus, in recent years HDACs have being leading focus for the clinical development of molecules that modulate their activities. A number of HDACi have been identified, they represent promising additive in cancer therapy as they can induce upregulation of specific proapoptotic genes and/or downregulation of prosurvival genes, therefore reactivating pathways controlling apoptosis, differentiation, or cell growth [27]. In recent in vitro studies, HDACi inhibitors such as TSA and SAHA have been shown to induce pancreatic cell death by apoptosis [60]. Other investigators have reported that TSA can synergize with gemcitabine [61] or the proteasome inhibitor PS-341 [62] to induce apoptosis of human pancreatic cancer cells. Thus, HDAC might also be promising targets for therapeutic intervention in pancreatic cancer.

With HDAC7 overexpression in pancreatic tumor tissues and not in their normal counterparts, it is reasonable to suggest that it might represent an interesting target for novel appraoches in the design of anti-pancreatic cancer therapies. However, rational targeting of HDAC7 signaling in this aggressive cancer disease needs further research to better understand its exact role in cancer progression. Also, it will require a clear understanding of cross-talk between HDAC7 signaling and other biochemical pathways that play a role in pancreatic cancer development. This may help to define which compound family could be more appropriate to be combined with HDAC7 inhibitors.

\section{Acknowledgments}

Dr. M. Ouaissi was supported by Assistance Publique Hôpitaux de Marseille and has received a grant from the NOVARTIS Laboratory.

\section{References}

[1] A. Jemal, R. Siegel, E. Ward, T. Murray, J. Xu, and M. J. Thun, "Cancer statistics, 2007," Ca-A Cancer Journal for Clinicians, vol. 57, no. 1, pp. 43-66, 2007.

[2] I. J. Fidler, "The pathogenesis of cancer metastasis: the 'seed and soil' hypothesis revisited," Nature Reviews Cancer, vol. 3, no. 6, pp. 453-458, 2003.

[3] L. Rosenberg, "Pancreatic cancer: a review of emerging therapies," Drugs, vol. 59, no. 5, pp. 1071-1089, 2000.

[4] M.-H. Kuo and C. D. Allis, "Roles of histone acetyltransferases and deacetylases in gene regulation," BioEssays, vol. 20, no. 8, pp. 615-626, 1998.

[5] J. E. Bolden, M. J. Peart, and R. W. Johnstone, "Anticancer activities of histone deacetylase inhibitors," Nature Reviews Drug Discovery, vol. 5, no. 9, pp. 769-784, 2006.

[6] R. A. Frye, "Phylogenetic classification of prokaryotic and eukaryotic Sir2-like proteins," Biochemical and Biophysical Research Communications, vol. 273, no. 2, pp. 793-798, 2000.

[7] F. De Nigris, J. Cerutti, C. Morelli et al., "Isolation of a SIRlike gene, SIR-T8, that is overexpressed in thyroid carcinoma cell lines and tissues," British Journal of Cancer, vol. 86, no. 6, pp. 917-923, 2002.

[8] A. J. M. De Ruijter, A. H. Van Gennip, H. N. Caron, S. Kemp, and A. B. P. Van Kuilenburg, "Histone deacetylases (HDACs): characterization of the classical HDAC family," Biochemical Journal, vol. 370, no. 3, pp. 737-749, 2003.

[9] J. E. Bradner, N. West, M. L. Grachan et al., "Chemical phylogenetics of histone deacetylases," Nature Chemical Biology, vol. 6, no. 3, pp. 238-243, 2010.

[10] C. M. Grozinger, E. D. Chao, H. E. Blackwell, D. Moazed, and S. L. Schreiber, "Identification of a class of small molecule inhibitors of the sirtuin family of NAD-dependent deacetylases by phenotypic screening," Journal of Biological Chemistry, vol. 276, no. 42, pp. 38837-38843, 2001.

[11] A. Bedalov, T. Gatbonton, W. P. Irvine, D. E. Gottschling, and J. A. Simon, "Identification of a small molecule inhibitor of Sir2p," Proceedings of the National Academy of Sciences of the United States of America, vol. 98, no. 26, pp. 15113-15118, 2001.

[12] J.-H. Choi, H. J. Kwon, B.-I. Yoon et al., "Expression profile of histone deacetylase 1 in gastric cancer tissues," Japanese Journal of Cancer Research, vol. 92, no. 12, pp. 1300-1304, 2001. 
[13] P. Zhu, E. Martin, J. Mengwasser, P. Schlag, K.-P. Janssen, and M. Göttlicher, "Induction of HDAC2 expression upon loss of APC in colorectal tumorigenesis," Cancer Cell, vol. 5, no. 5, pp. 455-463, 2004.

[14] A. J. Wilson, D.-S. Byun, N. Popova et al., "Histone deacetylase 3 (HDAC3) and other class I HDACs regulate colon cell maturation and p21 expression and are deregulated in human colon cancer," Journal of Biological Chemistry, vol. 281, no. 19, pp. 13548-13558, 2006.

[15] M. J. Scanlan, S. Welt, C. M. Gordon et al., "Cancer-related serological recognition of human colon cancer: identification of potential diagnostic and immunotherapeutic targets," Cancer Research, vol. 62, no. 14, pp. 4041-4047, 2002.

[16] F. De Nigris, J. Cerutti, C. Morelli et al., "Isolation of a SIRlike gene, SIR-T8, that is overexpressed in thyroid carcinoma cell lines and tissues," British Journal of Cancer, vol. 86, no. 6, pp. 917-923, 2002.

[17] M. Hiratsuka, T. Inoue, T. Toda et al., "Proteomics-based identification of differentially expressed genes in human gliomas: down-regulation of SIRT2 gene," Biochemical and Biophysical Research Communications, vol. 309, no. 3, pp. 558566, 2003.

[18] D. W. Kim, M. A. Sovak, G. Zanieski et al., "Activation of NF- $\kappa \mathrm{B} /$ Rel occurs early during neoplastic transformation of mammary cells," Carcinogenesis, vol. 21, no. 5, pp. 871-879, 2000.

[19] W. Weichert, M. Boehm, V. Gekeler et al., "High expression of RelA/p65 is associated with activation of nuclear factor- $\kappa \mathrm{B}$ dependent signaling in pancreatic cancer and marks a patient population with poor prognosis," British Journal of Cancer, vol. 97, no. 4, pp. 523-530, 2007.

[20] N. Mori, M. Fujii, S. Ikeda et al., "Constitutive activation of NF- $\kappa$ B in primary adult T-cell leukemia cells," Blood, vol. 93, no. 7, pp. 2360-2368, 1999.

[21] P. C. Cogswell, D. C. Guttridge, W. K. Funkhouser, and A. S. Baldwin Jr., "Selective activation of NF- $\kappa$ B subunits in human breast cancer: potential roles for NF- $\kappa \mathrm{B} 2 / \mathrm{p} 52$ and for Bcl-3," Oncogene, vol. 19, no. 9, pp. 1123-1131, 2000.

[22] B. P. Ashburner, S. D. Westerheide, and A. S. Baldwin Jr., "The p65 (RelA) subunit of NF- $\kappa$ B interacts with the histone deacetylase (HDAC) corepressors HDAC1 and HDAC2 to negatively regulate gene expression," Molecular and Cellular Biology, vol. 21, no. 20, pp. 7065-7077, 2001.

[23] L.-F. Chen, W. Fischle, E. Verdin, and W. C. Greene, "Duration of nuclear NF- $\kappa \mathrm{B}$ action regulated by reversible acetylation," Science, vol. 293, no. 5535, pp. 1653-1657, 2001.

[24] F. Yeung, J. E. Hoberg, C. S. Ramsey et al., "Modulation of NF- $\kappa$ B-dependent transcription and cell survival by the SIRT1 deacetylase," EMBO Journal, vol. 23, no. 12, pp. 2369-2380, 2004.

[25] M. Yoshida, M. Kijima, M. Akita, and T. Beppu, "Potent and specific inhibition of mammalian histone deacetylase both in vivo and in vitro by trichostatin A," Journal of Biological Chemistry, vol. 265, no. 28, pp. 17174-17179, 1990.

[26] M. S. Finnin, J. R. Donigian, A. Cohen et al., "Structures of a histone deacetylase homologue bound to the TSA and SAHA inhibitors," Nature, vol. 401, no. 6749, pp. 188-193, 1999.

[27] J. E. Bolden, M. J. Peart, and R. W. Johnstone, "Anticancer activities of histone deacetylase inhibitors," Nature Reviews Drug Discovery, vol. 5, no. 9, pp. 769-784, 2006.

[28] W. Xu, L. Ngo, G. Perez, M. Dokmanovic, and P. A. Marks, "Intrinsic apoptotic and thioredoxin pathways in human prostate cancer cell response to histone deacetylase inhibitor,"
Proceedings of the National Academy of Sciences of the United States of America, vol. 103, no. 42, pp. 15540-15545, 2006.

[29] A. Nebbioso, N. Clarke, E. Voltz et al., "Tumor-selective action of HDAC inhibitors involves TRAIL induction in acute myeloid leukemia cells," Nature Medicine, vol. 11, no. 1, pp. 77-84, 2005.

[30] A. Insinga, S. Monestiroli, S. Ronzoni et al., "Inhibitors of histone deacetylases induce tumor-selective apoptosis through activation of the death receptor pathway," Nature Medicine, vol. 11, no. 1, pp. 71-76, 2005.

[31] A. A. Ruefli, M. J. Ausserlechner, D. Bernhard et al., "The histone deacetylase inhibitor and chemotherapeutic agent suberoylanilide hydroxamic acid (SAHA) induces a cell-death pathway characterized by cleavage of Bid and production of reactive oxygen species," Proceedings of the National Academy of Sciences of the United States of America, vol. 98, no. 19, pp. 10833-10838, 2001.

[32] M. J. Peart, K. M. Tainton, A. A. Ruefli et al., "Novel mechanisms of apoptosis induced by histone deacetylase inhibitors," Cancer Research, vol. 63, no. 15, pp. 4460-4471, 2003.

[33] C. S. Mitsiades, N. S. Mitsiades, C. J. McMullan et al., "Transcriptional signature of histone deacetylase inhibition in multiple myeloma: biological and clinical implications," Proceedings of the National Academy of Sciences of the United States of America, vol. 101, no. 2, pp. 540-545, 2004.

[34] Y. Zhao, J. Tan, L. Zhuang, X. Jiang, E. T. Liu, and Q. Yu, "Inhibitors of histone deacetylases target the Rb-E2F1 pathway for apoptosis induction through activation of proapoptotic protein Bim," Proceedings of the National Academy of Sciences of the United States of America, vol. 102, no. 44, pp. 16090-16095, 2005.

[35] Y. Zhang, M. Adachi, R. Kawamura, and K. Imai, "Bmf is a possible mediator in histone deacetylase inhibitors FK228 and CBHA-induced apoptosis," Cell Death and Differentiation, vol. 13, no. 1, pp. 129-140, 2006.

[36] R. R. Rosato, J. A. Almenara, and S. Grant, "The histone deacetylase inhibitor MS-275 promotes differentiation or apoptosis in human leukemia cells through a process regulated by generation of reactive oxygen species and induction of p21CIP1/WAF1," Cancer Research, vol. 63, no. 13, pp. 36373645, 2003.

[37] P. García-Morales, A. Gómez-Martínez, A. Carrato et al., "Histone deacetylase inhibitors induced caspase-independent apoptosis in human pancreatic adenocarcinoma cell lines," Molecular Cancer Therapeutics, vol. 4, no. 8, pp. 1222-1230, 2005.

[38] M. S. Kim, H. J. Kwon, Y. M. Lee et al., "Histone deacetylases induce angiogenesis by negative regulation of tumor suppressor genes," Nature Medicine, vol. 7, no. 4, pp. 437-443, 2001.

[39] D. Z. Qian, Y. Kato, S. Shabbeer et al., "Targeting tumor angiogenesis with histone deacetylase inhibitors: the hydroxamic acid derivative LBH589," Clinical Cancer Research, vol. 12, no. 2, pp. 634-642, 2006.

[40] T. Maeda, M. Towatari, H. Kosugi, and H. Saito, "Upregulation of costimulatory/adhesion molecules by histone deacetylase inhibitors in acute myeloid leukemia cells," Blood, vol. 96, no. 12, pp. 3847-3856, 2000.

[41] W. J. Magner, A. L. Kazim, C. Stewart et al., "Activation of MHC class I, II, and CD40 gene expression by histone deacetylase inhibitors," Journal of Immunology, vol. 165, no. 12, pp. 7017-7024, 2000. 
[42] S. Zhao, K. Venkatasubbarao, S. Li, and J. W. Freeman, "Requirement of a specific Sp1 site for histone deacetylasemediated repression of transforming growth factor $\beta$ type II receptor expression in human pancreatic cancer cells," Cancer Research, vol. 63, no. 10, pp. 2624-2630, 2003.

[43] P. Reddy, Y. Maeda, K. Hotary et al., "Histone deacetylase inhibitor suberoylanilide hydroxamic acid reduces acute graft-versus-host disease and preserves graft-versus-leukemia effect," Proceedings of the National Academy of Sciences of the United States of America, vol. 101, no. 11, pp. 3921-3926, 2004.

[44] I. Nusinzon and C. M. Horvath, "Interferon-stimulated transcription and innate antiviral immunity require deacetylase activity and histone deacetylase 1," Proceedings of the National Academy of Sciences of the United States of America, vol. 100, no. 25, pp. 14742-14747, 2003.

[45] Z.-L. Yuan, Y.-J. Guan, D. Chatterjee, and Y. E. Chin, "Stat3 dimerization regulated by reversible acetylation of a single lysine residue," Science, vol. 307, no. 5707, pp. 269-273, 2005.

[46] A. Moldenhauer, R. C. Frank, J. Pinilla-Ibarz et al., "Histone deacetylase inhibition improves dendritic cell differentiation of leukemic blasts with AML1-containing fusion proteins," Journal of Leukocyte Biology, vol. 76, no. 3, pp. 623-633, 2004.

[47] C. Yu, M. Rahmani, D. Conrad, M. Subler, P. Dent, and S. Grant, "The proteasome inhibitor bortezomib interacts synergistically with histone deacetylase inhibitors to induce apoptosis in Bcr/Abl+ cells sensitive and resistant to STI571," Blood, vol. 102, no. 10, pp. 3765-3774, 2003.

[48] S. T. Nawrocki, J. S. Carew, M. S. Pino et al., "Aggresome disruption: a novel strategy to enhance bortezomib-induced apoptosis in pancreatic cancer cells," Cancer Research, vol. 66, no. 7, pp. 3773-3781, 2006.

[49] S. C. Maggio, R. R. Rosato, L. B. Kramer et al., "The histone deacetylase inhibitor MS-275 interacts synergistically with fludarabine to induce apoptosis in human leukemia cells," Cancer Research, vol. 64, no. 7, pp. 2590-2600, 2004.

[50] A. Jemal, T. Murray, E. Ward et al., "Cancer statistics, 2005," Ca-A Cancer Journal for Clinicians, vol. 55, no. 1, pp. 10-30, 2005.

[51] H. A. Burris III, M. J. Moore, J. Andersen et al., "Improvements in survival and clinical benefit with gemcitabine as firstline therapy for patients with advanced pancreas cancer: a randomized trial," Journal of Clinical Oncology, vol. 15, no. 6, pp. 2403-2413, 1997.

[52] M. J. Moore, D. Goldstein, J. Hamm et al., "Erlotinib plus gemcitabine compared with gemcitabine alone in patients with advanced pancreatic cancer: a phase III trial of the National Cancer Institute of Canada Clinical Trials Group," Journal of Clinical Oncology, vol. 25, no. 15, pp. 1960-1966, 2007.

[53] H. L. Kindler, D. Niedzwiecki, D. Hollis et al., "A double-blind, placebo-controlled, randomized phase III trial of gemcitabine (G) plus bevacizumab (B) versus gemcitabine plus placebo (P) in patients (pts) with advanced pancreatic cancer (PC): a preliminary analysis of Cancer and Leukemia Group B (CALGB)," Journal of Clinical Oncology, vol. 25, no. 18S, article 4508, 2007.

[54] P. A. Philip, J. Benedetti, C. Fenoglio-Preiser et al., "Phase III study of gemcitabine plus cetuximab versus gemcitabine in patients with locally advanced or metastatic pancreatic adenocarcinoma: SWOG S0205 study," Journal of Clinical Oncology, vol. 25, article LBA4509, 2007.

[55] E. K. Rowinsky, J. J. Windle, and D. D. Von Hoff, "Ras protein farnesyltransferase: a strategic target for anticancer therapeutic development," Journal of Clinical Oncology, vol. 17, no. 11, pp. 3631-3652, 1999.

[56] E. Van Cutsem, H. Van De Velde, P. Karasek et al., "Phase III trial of gemcitabine plus tipifarnib compared with gemcitabine plus placebo in advanced pancreatic cancer," Journal of Clinical Oncology, vol. 22, no. 8, pp. 1430-1438, 2004.

[57] J. Bai, J. Sui, A. Demirjian, C. M. Vollmer Jr., W. Marasco, and M. P. Callery, "Predominant Bcl-XL knockdown disables antiapoptotic mechanisms: tumor necrosis factor-related apoptosis-inducing ligand-based triple chemotherapy overcomes chemoresistance in pancreatic cancer cells in vitro," Cancer Research, vol. 65, no. 6, pp. 2344-2352, 2005.

[58] N. Bardeesy and R. A. DePinho, "Pancreatic cancer biology and genetics," Nature Reviews Cancer, vol. 2, no. 12, pp. 897909, 2002.

[59] M. Cascalló, J. Calbó, G. Capellà, C. Filiat, M. Pastor-Anglada, and A. Mazo, "Enhancement of gemcitabine-induced apoptosis by restoration of p53 function in human pancreatic tumors," Oncology, vol. 68, no. 2-3, pp. 179-189, 2005.

[60] P. García-Morales, A. Gómez-Martínez, A. Carrato et al., "Histone deacetylase inhibitors induced caspase-independent apoptosis in human pancreatic adenocarcinoma cell lines," Molecular Cancer Therapeutics, vol. 4, no. 8, pp. 1222-1230, 2005.

[61] M. Donadelli, C. Costanzo, S. Beghelli et al., "Synergistic inhibition of pancreatic adenocarcinoma cell growth by trichostatin A and gemcitabine," Biochimica et Biophysica Acta, vol. 1773, no. 7, pp. 1095-1106, 2007.

[62] J. Bai, A. Demirjian, J. Sui, W. Marasco, and M. P. Callery, "Histone deacetylase inhibitor trichostatin A and proteasome inhibitor PS-341 synergistically induce apoptosis in pancreatic cancer cells," Biochemical and Biophysical Research Communications, vol. 348, no. 4, pp. 1245-1253, 2006.

[63] M. Ouaïssi, S. Cabral, J. Tavares et al., "Histone deacetylase (HDAC) encoding gene expression in pancreatic cancer cell lines and cell sensitivity to HDAC inhibitors," Cancer Biology and Therapy, vol. 7, no. 4, pp. 523-531, 2008.

[64] M. Ouaïssi, I. Sielezneff, R. Silvestre et al., "High histone deacetylase 7 (HDAC7) expression is significantly associated with adenocarcinomas of the pancreas," Annals of Surgical Oncology, vol. 15, no. 8, pp. 2318-2328, 2008.

[65] B. Gerdes, A. Ramaswamy, A. Ziegler et al., "p16INK4a is a prognostic marker in resected ductal pancreatic cancer: an analysis of p16INK4a, p53, MDM2, an Rb," Annals of Surgery, vol. 235, no. 1, pp. 51-59, 2002.

[66] D. Mottet, A. Bellahcène, S. Pirotte et al., "Histone deacetylase 7 silencing alters endothelial cell migration, a key step in angiogenesis," Circulation Research, vol. 101, no. 12, pp. 12371246, 2007.

[67] P. A. Marks and R. Breslow, "Dimethyl sulfoxide to vorinostat: development of this histone deacetylase inhibitor as an anticancer drug," Nature Biotechnology, vol. 25, no. 1, pp. 8490, 2007.

[68] M. Dokmanovic, G. Perez, W. Xu et al., "Histone deacetylase inhibitors selectively suppress expression of HDAC7," Molecular Cancer Therapeutics, vol. 6, no. 9, pp. 2525-2534, 2007.

[69] L. Stimson and N. B. La Thangue, "Biomarkers for predicting clinical responses to HDAC inhibitors," Cancer Letters, vol. 280, no. 2, pp. 177-183, 2009.

[70] A. Manuyakorn, R. Paulus, J. Farrell et al., "Cellular histone modification patterns predict prognosis and treatment response in resectable pancreatic adenocarcinoma: results from RTOG 9704," Journal of Clinical Oncology, vol. 28, no. 8, pp. 1358-1365, 2010. 
[71] F. Dequiedt, H. Kasler, W. Fischle et al., "HDAC7, a thymusspecific class II histone deacetylase, regulates Nur77 transcription and TCR-mediated apoptosis," Immunity, vol. 18, no. 5, pp. 687-698, 2003.

[72] J. Chen, W. Fiskus, K. Eaton et al., "Cotreatment with BCL2 antagonist sensitizes cutaneous T-cell lymphoma to lethal action of HDAC7-Nur77-based mechanism," Blood, vol. 113, no. 17, pp. 4038-4048, 2009.

[73] H.-Y. Kao, A. Verdel, C.-C. Tsai, C. Simon, H. Juguilon, and S. Khochbin, "Mechanism for nucleocytoplasmic shuttling of histone deacetylase 7," Journal of Biological Chemistry, vol. 276, no. 50, pp. 47496-47507, 2001.

[74] W. Fischle, F. Dequiedt, M. Fillion, M. J. Hendzel, W. Voelter, and E. Verdin, "Human HDAC7 histone deacetylase activity is associated with HDAC3 in vivo," Journal of Biological Chemistry, vol. 276, no. 38, pp. 35826-35835, 2001.

[75] S. B. Baylin and J. E. Ohm, "Epigenetic gene silencing in cancer-a mechanism for early oncogenic pathway addiction?” Nature Reviews Cancer, vol. 6, no. 2, pp. 107-116, 2006.

[76] P. P. Pandolfi, "Histone deacetylases and transcriptional therapy with their inhibitors," Cancer Chemotherapy and Pharmacology, vol. 48, no. 1, pp. S17-S19, 2001. 

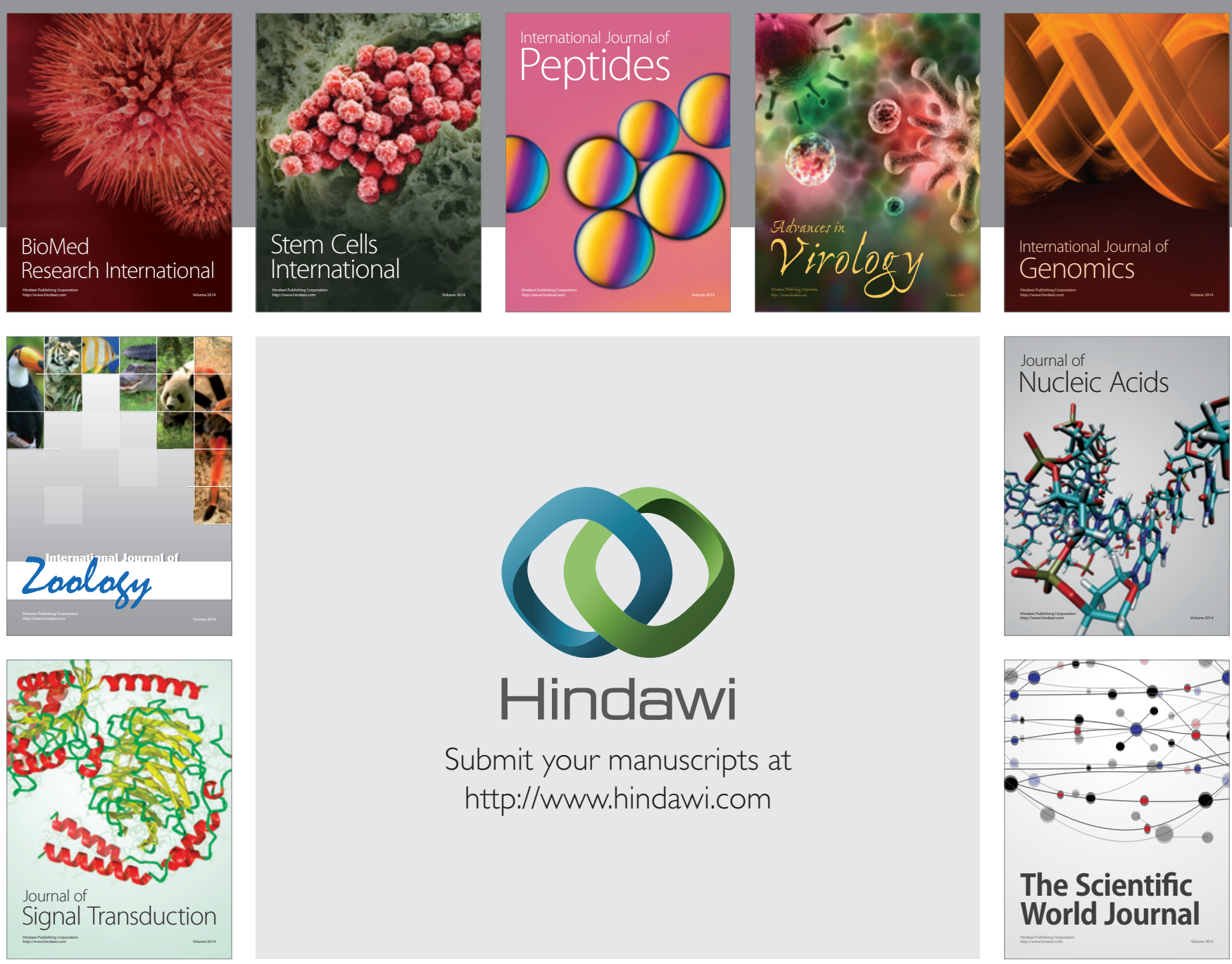

Submit your manuscripts at

http://www.hindawi.com
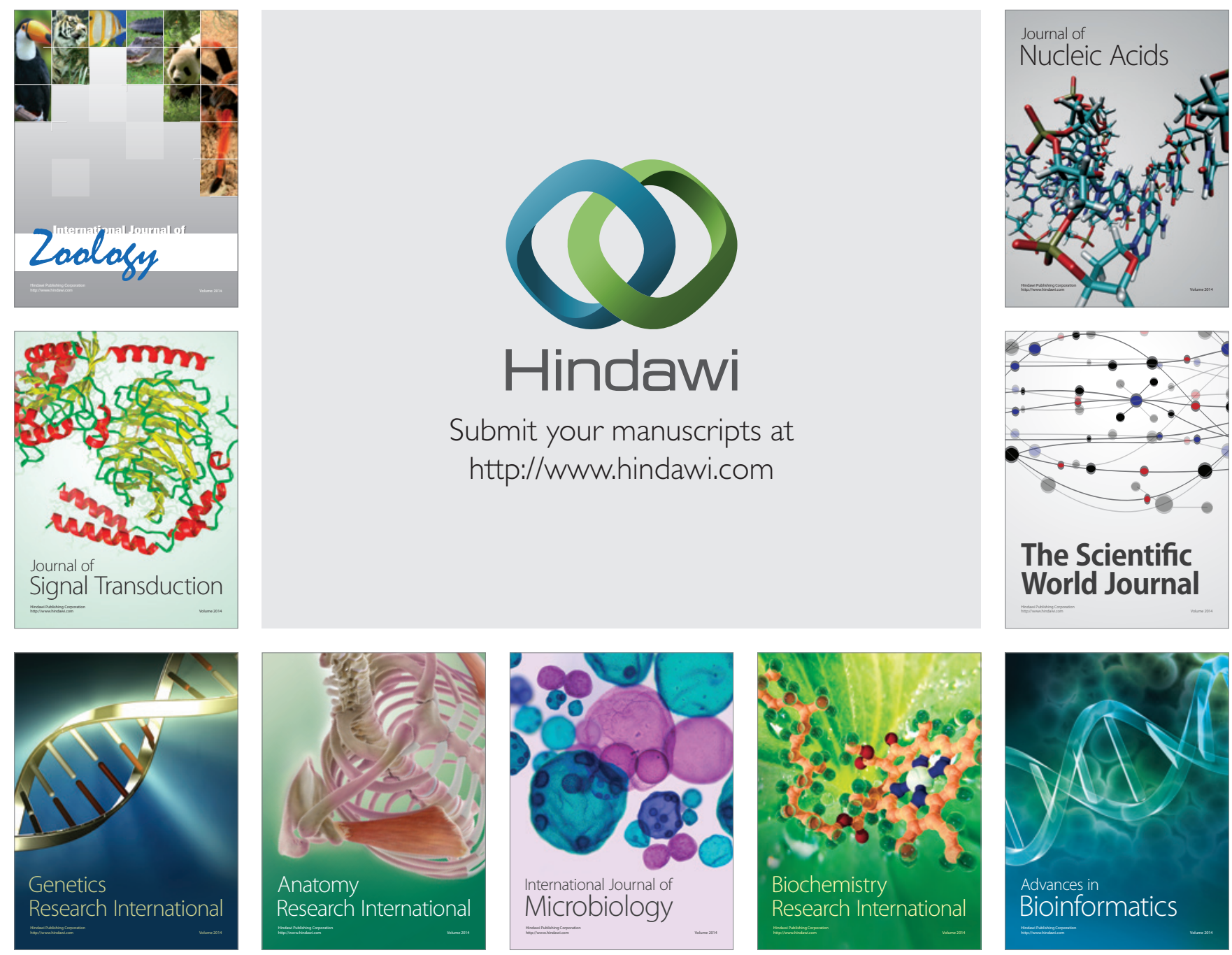

The Scientific World Journal
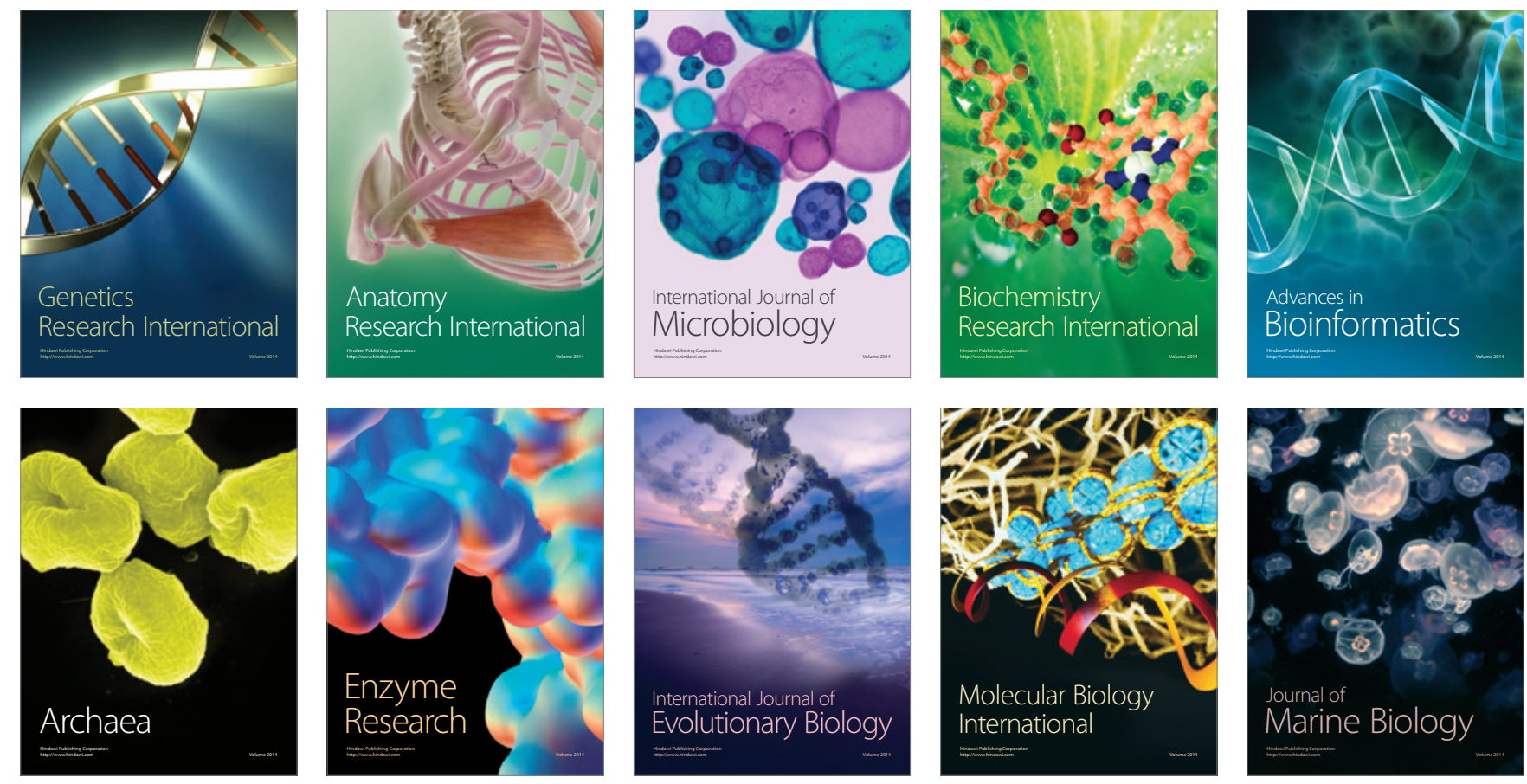